\title{
Correction to: Rank and Pseudo-Rank Procedures for Independent Observations in Factorial Designs
}

\section{Correction to:}

E. Brunner et al., Rank and Pseudo-Rank Procedures for Independent Observations in Factorial Designs, Springer Series in Statistics, https://doi.org/10.1007/978-3-030-02914-2

This book was inadvertently published with an incorrect affiliation of the Author Frank Konietschke.

The correct affiliation is:

Frank Konietschke

Institute of Biometry and Clinical Epidemiology

Charité - University Medical School

Berlin, Germany

The updated online version of the book can be found at https://doi.org/10.1007/978-3-030-02914-2 